\title{
A RESUME OF MY YEAR'S WORK WITH SUSPENSION LARYNGOSCOPY.*
}

\author{
Dr. Robis't Clydr Lrinch, New Orleans, La.
}

Last year you conferred on me the signal honor of being permitted to demonstrate to you suspension laryngoscopy as I practice it, to detail for you the various reasons for the modifications which secmed to me improvements over the instrument used by Killian.

In my contributions on this strbject, up to this time I have clealt with the technique of acquiring an unobstructed view of the laryix, including the anterior commissure and in most instances a small area just superior to it, this field being sufficiently broad to enable one to practice the new type of intra-laryngeal surgery.

I will now devote the time you so generously allowed me to reviewing my work in this new two-handed surgery of the larynx. The term, while not thoroughly scientific, is descriptive and at once differentiates it from all the preceding methods. It may be in keeping lere for me to say that I have not found it necessary to change the model of the instrument in any way since its presentation last year, and have added to it only in three ways: two of these because of two accidents which must be made known to you now that you may provide against such occurrences in your work.

In a case being suspended for malignancy, a man of 68 , well preserved and weighing about $1^{\text {m }} 55$ pounds, with what appeared to be good, solidly fixed teeth, the view seemed a little difficult to acquire. A little more lifting and a little jacking of the epiglottis brought the tumor well into view. A section was removed for immediate pathological diagnosis when suddenly the instrument slipped from the patient's mouth. The cause of this was a fracture of the alveolus carrying his five front teeth of the upper jaw, the mass hanging from the mucous membrane on the labial aspect. The spatula was quickly removed, the teeth wired back in place with silver wires and I an glad to report they are now firmly rooted, healthy and apparently as good as ever. To counteract this accident I now place this strap under the occiput of the patient and clamp it into the angles of the pear-shaped ring. By tightening it sufficiently, one relieves almost completely the pressure of the tooth plates against the teeth and it will be impossible for

\footnotetext{
*Read before the American Laryngological Association, Washington, D. C., May 10, 1016.
} 
this accident to happen again. The other accident resulted from the tongue pattula breaking at its angle. As this spatula was the first made by the instrument maker its construction was not so perfect as it now is. The danger here lay in the sudden dropping of the patient's head from the spatula and a possible fracture of the cervical vertebra. 'I'o counteract this I have incorporated in my new suspension table this feature of having the head piece drop at various angles so that should the patient drop for any reason the support would be in place to receive the head.

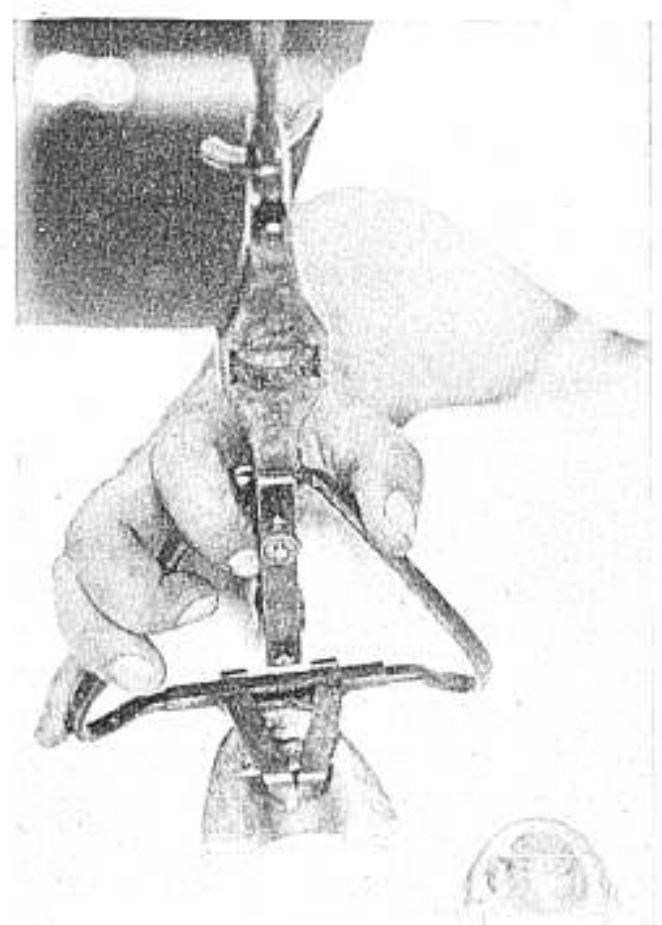

Figuro 1. To illustrate the dental spoons (two sizes) and the toothplates fiting in the horizontal flled-in face.

While reviewing my cases operated on from one to two years ago, I noticed in two or three of the adult cases the loss of the enamel of the teeth from contact with the tooth plate or plates. After further observation this I found to be due to the fact that there was a lateral fracture of the teeth, caused by the pressure downward and outward of the tooth plates of the mouth gag. In a few instances I have observed the loss of the enamel of the teeth on the biting edge. It also happened that in patients whose teeth were very irregular and in some instances where carious tooth 
roots happened to be present or where the only teeth available to fix the tooth plates were loose, decayed or painful, that it became quite a problem to find a means of adjusting the tooth plates so as to do the least harm.

I have now surmounted these difficulties most satisfactorily by using dental impression spoons filled with moulding compound and make an impression of the entire upper jaw including the hard palate. When cold this impression must naturally fit accurately the entire upper jaw and if a tooth is loose or the gum painful in any particular spot the compound can be cut away so as to ease the pressure on this particular locality. In order that the tooth plates should now hold firmly against the smooth surface of the spoon, I have found it necessary to fill in the concavity in such a way as to give a square surface of sufficient depth for the tooth plates to gain firm hold. 'This has necessitated the lengthening of the tooth plates considerably and changing their shape in order to preserve the base of the fixing triangle.

Now that I have used this device a few times I find it far superior to the tooth plates in every way. It now offers complete [rotection to the teeth, absolutely equalizing the pressure upon the oral surface of the upper jaw. It will protect sensitive areas and loose teeth and I have no fear for the irregularity of the teeth or their condition. No more fractures of the enamel occur and the localized pain that was complained of previously is now no longer felt. It markedly facilitates the introduction of the spatula and makes it easier to keep the spatula in the middle line. Since the tooth plates have such a firm grip on the metal surface and such a broad area from pressure downward, there is much less danger of losing the view after it is once obtained. This to me is one of the best additions I have made for some time and I recommend it to you most heartily. Two sizes, one for adults and one for children, seem so far all that are necessary, and are designed to fit the one set of tooth plates.

'The table that I am using is one constructed from an old high-low dental base which will permit the table to be lowered or raised twenty inches. This, you see, permits the patient, in suspension, being raised to the proper height for the comfort of the operator, thus facilitating his work. The table has various other features which may be of interest. The top is of solid maple, one and one-half inches thick, because it forms a very firm support for the traveling crane and is itself thick enough to permit of perfect rigidity. The top can be 
tilted at various angles, raising or lowering the head to an angle of forty-five degrees; the top can be moved in a circle to take advantage of any light, and lastly, one can, if he chooses, perform the turning test for the labyrinth with the patient lying on the table. Foot and shoulder braces are so arranged as to fix the patient both comfortably and rigidly. The shoulder braces act effectually in preventing the patient from slipping up on the table while under heavy extension and although it is primarily designed for suspension work, it is a most serviceable ear, nose and throat operating table.

In my work I have discarded all the accessory illuminating appliances, except the deep tracheal searcher, and confine myself to an ordinary head mirror and a nitrogen lamp. The latter is always

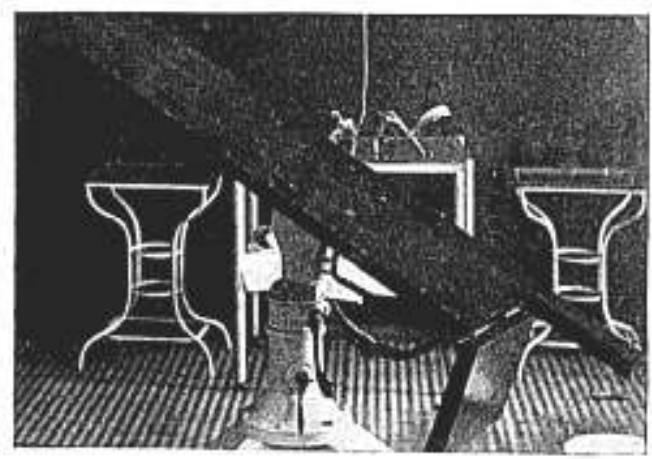

Figure 2. Table showing Lynch top with head lowered. Top can be lifted as far in the opposite direction.

ready, always works and is after all under the best control of the operator.

Intrinsic epithelioma of larynx. Let it be distinctly understood that I do not propose the removal of the intrinsic malignant growths of the larynx under suspension as a substitute for or advise it as better than thyrotomy, for the special reason that my own experience covers too few cases and my work is too young to be of deciding value at this time. I propose, however, to state to you my experience so that you may discuss my views and that we may establish a careful foundation upon which to work.

Mr. B. was operated on May 1, 1913. He has remained perfectly well up to this time and seems now to be in good shape. In the early part of 1914, he seemed to have a small mass at the anterior commissure which was removed and found to be only scar tissue. The tumor was removed completely and an immediate 
section made at the time and since then. The tumor showed the characteristics of malignancy.

H. W. was operated in July, 1914. $\mathrm{He}$ is still well and has resumed his occupation of vaudeville singer; with the exception that his voice has changed from a good baritone to a fair tenor. In him malignancy, microscopically and clinically, was unmistakable

Four cases were operated on in 1915. Three of these are wei and without evidence of recurrence. The fourth is a bit interesting. Mr. H. was referred to me because of papilloma. Clinically the diagnosis was suspicious of malignancy. Fixation of the arytenoid was present, radiating pains to the ear of the same side, continued hoarseness for one year. Age 38. At operation under suspension a specimen was removed for microscopic rush diagnosis, reported pipilloma and no malignancy as I have most profound confidence in our pathologist. I accordingly began a dissection of the mass and while it seemed at the time as though the larynx was clear, there still remained an indurated base which felt to me different from the preceding papilloma cases. In six weeks from the date of suspension there was a marked recurrence requiring tracheotomy and a few days later thyrotomy. At this time it was seen that the mass was infiltrating the posterior half of the larynx and there was suspicion of it having crossed the anterior commissure. Though I was not entirely satisfied with the result, we had no permission to remove the larynx as completely as possible with the thyrotomy. In three months the patient submitted to a laryngectomy and is now in most excellent health, working hard and apparently enjoying life in this altered state.

May I here repeat my advice that where one has malignancy of the larynx involving its posterior half with fixation of the joint and slight thickening or edema over the arytenoid, such a case, even though intrinsic, is unfit for operation by suspension. Under suspension one can make such an examination of the larynx as to determine an extension downward to the crico-thyroid membrane. In one case, not included in this list, we determined, by the direct method, the mass to be entirely intrinsic; no cervical glands were enlarged and we could not palpate anything to be construed as extension downward. Under suspension we could see distinctly the extension downward to the crico-thyroid membrane and in order to be certain of the position of this area, I put a fine hypodermic needle through the crico-thyroid membrane externally and used this as a land-mark. It was a revelation to see the mass beyond the needle, clipping or dimpeling into the crico-thyroid space. The pa- 
tient underwent a laryngectomy and has resumed his occupation of fishing and is working in comfort.

Four cases have been operated on so far this year. 'They are well. I mean by this that they healed and returned home to report again at intervals of three months. The fourth lost his front teeth, as before mentioned, and is now tunder radium, to be suspended again if the latter does not bring about the desired result.

I may mention again that dissection under suspension is not difficult and can be clone without permitting an instrument to touch the tumor mass. It can include the perichondrum, does not require much time, there is no excessive bleeding, does not require the

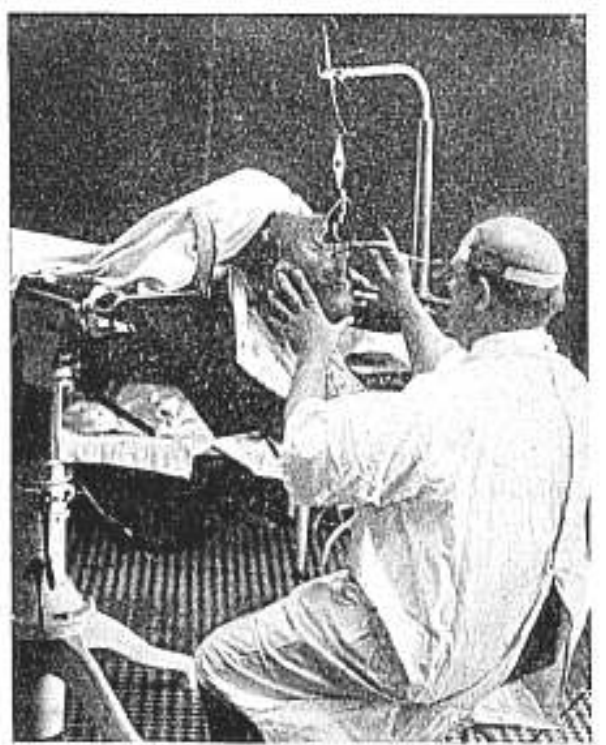

Figure 3. Operating in sitting position, showing top raised to proper height and shoulder brace support under head properly placed to prevent accident if patlent should drop from crune.

opening of the cartilagenous box, and is not in consecluence followed by stenosis. None of my cases have had any post-operative disturbance and they are usually up and out as soon as the ether is exhaled. 'There has been no shock so far and a most surprising lack of reaction.

Non-malignant tumors. Papilloma. I have removed by dissection multiple papilloma from the larynx in cases ranging from 18 months to 16 years, with no recurrence in a single case so far. The shortest time since operation is five months, the longest three years. In the case reported to you last year during the discussion 
of Dr. Hubbard's paper, the recurrence was beneath the cord, an entirely new site from that originally operated on and it followed an attack of whooping cough one year after the operation.

Single pedunctlated papilloma and those growing from broad bases have been operated on with no recurrence to date, the cases numbering nineteen in all.

Pick the mass up and dissect it well below the base from which it grows; to be more certain curette this base, paint the area with purc alcohol and dress the wound with tincture of benzoin com pound.

Pednnculated fibroma, growing from the loose tissue beneath the cord by a fairly large pedicle was removed by dissection and the wound stutured, healed by first intention. No recurrence.

Vocal nodules. Only those are selected for operation that cannot be removed by medical or contributory surgical procedures. Three cases; one congenital, two in school teachers; all symptomatically well. The school teachers have been referred to singing teachers for instruction in voice use and have remained well.

Cyst of the aryteno-epiglottic fold. About the size of a marble three-fourths of an inch in diameter was dissected without rupture and the wound stitched with good union and complete recovery. To remove the sack entirely is the classical surgery of cysts. It cannot be done with one hand.

Pachydermic laryngitis. Two cases both males, farmer and preacher, 30 and 40 years old respectively. In the former there was a hypertrophic mass at vocal process and extending along the free border of the right arytenoid cartilage; mass remoyed by wedge incision and free edge stitched with two sutures. Healing rapid and the result perfect. In the latter, much thickened inter-aryte- noid space was much improved by three horizontal deep cauterizations with actual cautery. It was very simple and accurate to apply the cautery in this case and an easy matter to protect the surrounding structures from burning. Such procedure would be impossible without suspension.

Perichondritis of thyroid. Patient C. D., 20 years old, cook by occupation. Followed by abscess formation. Patient suffered intense pain upon swallowing due to the inflammatory process and dyspnea from swelling and edema of the glottis. It seemed that tracheotomy would be immediately necessary. The patient was suspended and by palpating over the swollen surface with a cotton tipped applicator a soft fuctuating area was discovered; an aspirating needle was introduced and pus obtained. Free incision was 
made and the opening dilated with artery forceps after the method of Hilton. Catgut strands were placed in the incision to maintain the opening. A rapid subsidence of the swelling followed; patient was able to take nourishment freely and made a good recovery with a much disorganized larynx. At the time of the final visit one could not recognize any cords, false or true, and what was larynx was now a tube lined with scar tissue through which the patient breathed.

Fracture of thyroid cartilage. C. G., 11 years old, while at school, received a blow on the left side of the lateral cervical region

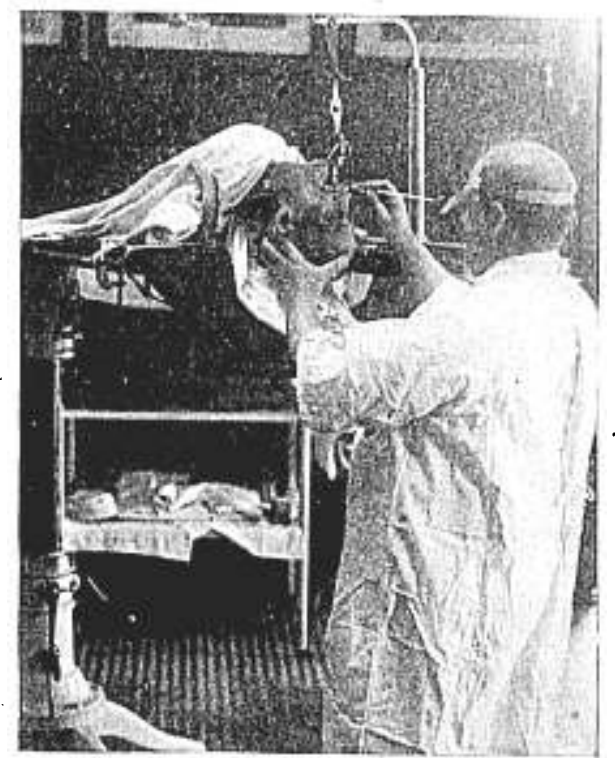

Figure 4. Operating in standing position. Table raised to full height of 54 inches.

and suffered at once from dyspnea, loss of voice, pain in the region of larynx aggravated on swallowing. On admission it was impossible to see the larynx and an $x$-ray showed a transverse fracture of the thyroid cartilage about its middle.

Under anesthesia, crepitus could be determined and with the aid of suspension we could see swelling on the left lateral half of the larynx with marked tumefaction about the region of the true cord. By manipulation we were able to reduce the fracture which reestablished the larynx to its normal lumen. A large intubation tube was then introduced as a splint to maintain the fragments. This remained in place about two weeks when it was coughed out 
and the larynx was seen to maintain its usual shape. About four weeks later the patient returned with marked dyspnea, due, this time, to granulations growing into the larynx presumably from the time of fracture. 'There was also quite an area of redunclent tissue over the upper surface of the arytenoids which was drawn into the larynx with each inspiration. Under suspension the granulation tissue was removed and its base cauterized with trichloracetic acid. 'The redundent tissue was actually removed by dissection and the raw stufaces stitched, requiring three sutures on each side. Healing was entirely satisfactory but the patient maintained an aphonic

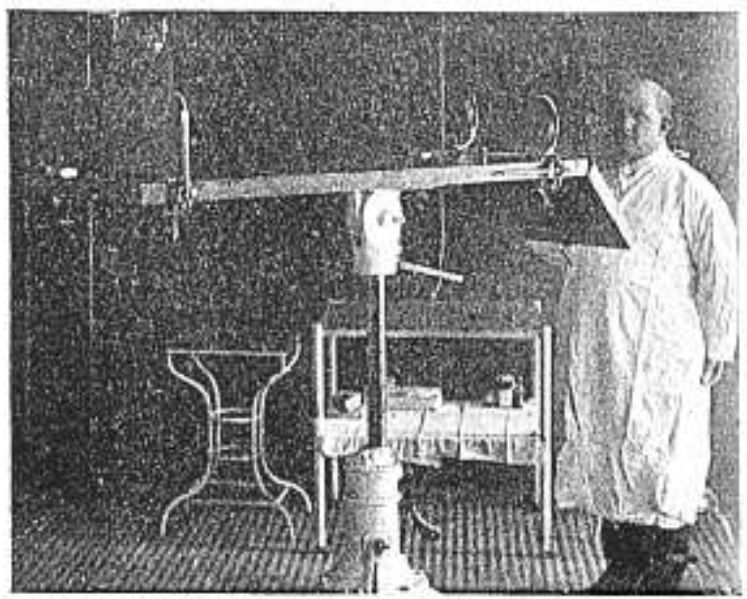

Figure 5. Table at full height with shoulder and foot braces.

voice for some time after this procedture, though under the anesthetic he cried out with a good tone. He has since regained his voice. The successful termination of this case was due in a very great measure to the suspension apparatus.

Ulcerative tubercular laryngitis. Six cases have been cauterized; in two, over three-fourths of the area of the larynx. 'There was very marked relief from pain. Healing followed in all of the cases. This, however, was only temporary, for all of the cases were of the third stage with progressive pulmonary destruction.

Abscess of epiglottis. The lingual surface of the epiglottis was the seat of accumulation of pus. Incision and drainage was very easy under suspension.

Pedunculated epithcliona. The mouth of the esophagus was the seat of lesion in my two cases. A negro 80 years old, was suspended under cocain anesthesia, the mass resected and five stitches 
placed. Healing was complete by primary union. Recurrence in ten months. At the site of removal, recurrence was by a rather broad and flat uleer about the size of a quarter dollar on the posthypopharyngeal wall. The mass was catuterized after the technicue of Percy, using a heavy clectro-catutery point. Much swelling and discomfort followed. 'Jhe patient has remained well with no recurrence up to this time.

the second case was in a white male. 'l'he mass sprung from the posterior lateral aspect of the cricoid cartilage and the lateral wall of the esophagus. 'The mass was removed and the base completely catuterized. The pationt's life was prolonged about ten months.

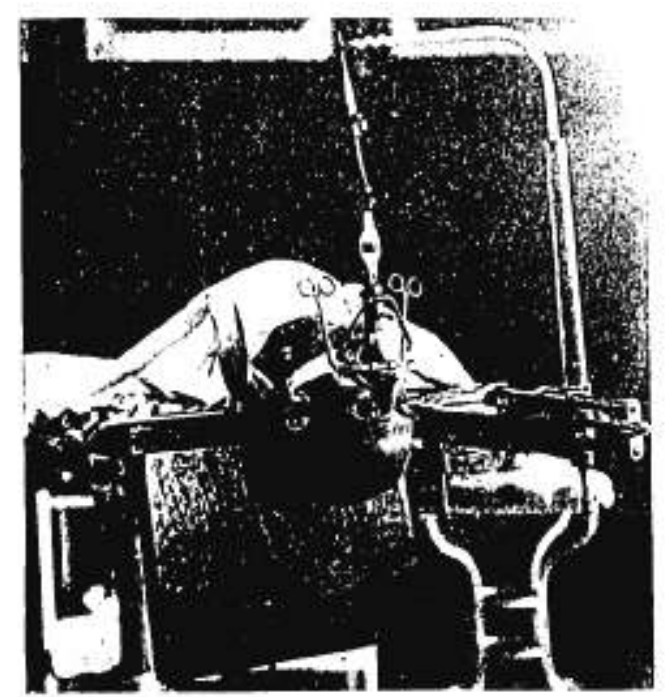

Fisure 6. Strap around head and clamped into ring on hook to prevent outward pressure on teeth and to insure steadiness of instruments.

This technique requires two free hands and is only possible under suspension and it may hold for us some hope in the fight against these lesions in this locality.

Forcign bodics in trachea and csophagus. In babies the suspension apparatus permits the careful passage of the tube beyond the vocal cords and subglottic space which it is so necessary not to traumatize. I have removed waternelon seeds from the trachea of children without the use of a tube, and a peanut impacted in the right main bronchus under suspension with the suction tube and no forceps. It was very easy to see the foreign body and to apply the tube. A safety pin with the point up, a jack stone and 
a quarter were found in children from eleven months to two years of age. The foreign bodies were removed very nicely from the upper end of the esophagus. While this is quite possible by the usual methods, one does the work more carefully, with less traumatism and with more exactness than by the usual methods. With two hands free a part can be lifted, points protected, bodies rotated, the cricoid lifted and moved from side to side in order to permit dislodgment and extraction without tearing the mucous membrane or otherwise injuring the parts. It also facilitates the introduction of the esophagoscope in certain cases.

I prefer, however, when working with an esophagoscope for a bronchoscope to introduce the tubes tunaided. If suspension is necessary for their introduction the patient should be relieved from the suspension apparatus after the tube has been passed so that subseculuent manipulation will not be interfered with

634: Maison Blanche Building.

The Treatment of Epithelioma of the Lower Lip. RUSSEL $H$. BogGs, Interstate Med: Jour., May, 1916.

As experience has taught that an epithelioma of the lower lip is rather a regional than a local lesion, surgical removal, wide and radical, has proved inefficient because a recurrence takes place in. over 50 per cent of cases when there are no palpable glands at the time of operation, and in over 75 per cent of cases when glandular involvement is present. 'The author believes that radiotherapy (meaning radium and the Roentgen rays) will give better results than surgery and surgical removal should only be done in selected cases. The technique, when using radium or the Roentgen rays, must be varied somewhat for the individual case. One advantage of radium is that it produces a more intense reaction, which disappears much more rapidly than a reaction of the same degree produced by the Roentgen rays. Before a case is considered clinically cured the resulting scar must be healthy, pliable, without any retraction and without scaliness. The treatment of the adjacent glands is most important and should never be omitted, no matter how small the lesion.

P. F. 